\title{
ORIGINAL ARTICLE Systemic hypothermia in acute cervical spinal cord injury: a case-controlled study
}

\author{
M Dididze, BA Green, W Dalton Dietrich, S Vanni, MY Wang and AD Levi
}

Introduction: Systemic hypothermia remains a promising neuroprotective strategy. There has been recent interest in its use in patients with spinal cord injury $(\mathrm{SCl})$. In this article, we describe our extended single center experience using intravascular hypothermia for the treatment of cervical SCI.

Methods: Thirty-five acute cervical SCl patients received modest $\left(33^{\circ} \mathrm{C}\right)$ intravascular hypothermia for $48 \mathrm{~h}$. Neurological outcome was assessed by the International Standards for Neurological Classification of Spinal Cord Injury scale (ISNCSCI) developed by the American Spinal Injury Association. Local and systemic complications were recorded.

Results: All patients were complete ISNCSCI A on admission, but four converted to ISNCSCI B in $<24$ h post injury. Hypothermia was delivered in 5.76 ( \pm 0.45 ) hours from injury if we exclude four cases with delayed admission ( $>18 \mathrm{~h}$ ). Fifteen of total 35 patients (43\%) improved at least one ISNCSCI grade at latest follow up 10.07 ( \pm 1.03 ) months. Even excluding those patients who converted from ISNCSCI A within $24 \mathrm{~h}, 35.5 \%$ (11 out of 31 ) improved at least one ISNCSCI grade. Both retrospective $(n=14)$ and prospective $(n=21)$ groups revealed similar number of respiratory complications. The overall risk of any thromboembolic complication was $14.2 \%$.

Conclusion: The results are promising in terms of safety and improvement in neurological outcome. To date, the study represents the largest study cohort of cervical SCI patients treated by modest hypothermia. A multi-center, randomized study is needed to determine if systemic hypothermia should be a part of SCI patients' treatment for whom few options exist.

Spinal Cord (2013) 51, 395-400; doi:10.1038/sc.2012.161; published online 18 December 2012

Keywords: acute cervical SCl; cooling catheter; systemic hypothermia

\section{INTRODUCTION}

Hypothermia continues to show promise in a variety of acute central nervous system injuries. Various factors need to be considered with systemic cooling of the spinal cord injury (SCI) patient, including methods of cooling, window from injury to initiation, duration and depth of hypothermia, and rate of re-warming. Two main methods of spinal cord cooling exist. Local cooling (epidural vs intradural) has a rich history with both experimental and clinical evidence supporting its use. ${ }^{1}$ Systemic cooling may be applied either via a transcutaneous or intravascular route. Modern cooling blankets can be applied and have the advantage of being less invasive but also the disadvantage of being less precise with regards to temperature control. ${ }^{2}$ While profound levels of hypothermia $\left(\mathrm{T}<32{ }^{\circ} \mathrm{C}\right)$ can be difficult to administer and are subject to increased complication rates, mild (modest) levels of hypothermia $\left(\mathrm{T} 32-34{ }^{\circ} \mathrm{C}\right.$ ) have been shown to provide significant protection against traumatic and ischemic neuronal cell death. ${ }^{2,3}$ When administered after experimental acute SCI, there is a reduction in the volume of histopathological damage and a concomitant improvement in BBB walking index. ${ }^{4,5}$ A recent study revealed beneficial effect of hypothermia on somato-sensory and motor-evoked potentials. ${ }^{6}$ Our reported experience of 14 cervical SCI patients revealed that systemic hypothermia induced via an intravascular catheter and continued for $48 \mathrm{~h}$ showed improved functional outcome and appeared safe compared with a historical control group. ${ }^{7,8}$ In this article we describe a larger series of cervical SCI patients based on our extended, single center experience of hypothermia treatment that now spans over 6 years.

\section{MATERIALS AND METHODS}

We present 35 acute cervical SCI patients, who received modest hypothermia in our institution: 14 patients were retrospectively analyzed (April 2006 to June 2008) and 21 were prospectively evaluated (May 2009 to April 2012) using University of Miami institutional review board-approved hypothermia protocols (\# 20071018 and \# 20060556, respectively). Patients were admitted to the Emergency Department at the Ryder Trauma Center of Jackson Memorial Hospital. Initial neurological examinations and clinical outcome at follow-ups were completed according to American Spinal injury Association and International Medical Society of Paraplegia Impairment Scales (ISNCSCI). During the neurological assessment, patients were not under the influence of sedatives, muscle relaxants or alcohol/drug intoxication and did not have head trauma. Patients with an admission ISNCSCI A Glasgow Coma Scale of 15, 18-65 years of age and non-penetrating cervical injury were included into hypothermia protocol. Patients with hyperthermia on admission $\left(\mathrm{T}>38.5^{\circ} \mathrm{C}\right.$ ), severe systemic injury, coagulopathy, thrombocytopenia, pancreatitis, Reynaud's syndrome, severe bleeding, pregnancy, known prior cardiac history, and who were intubated and sedated before initial neurological examination were excluded (Table 1). No patient received either steroids or any other neuroprotective drug. Hypothermia administration did not affect the decision for timing of cranial tong application and reduction or surgical intervention. To deliver intravascular hypothermia, an Alsius Icy CoolGuard 
Table 1 Inclusion/exclusion criteria for initiation of therapeutic hypothermia

\begin{tabular}{ll}
\hline Inclusion criteria & Exclusion criteria \\
\hline $18-65$ years of age ISNCSCI Score & Age $>65$ years \\
A GCS = 15 Non-penetrating injury & ISNCSCI Score B, C or D \\
Patients urgently taken to the oper- & Hyperthermia on admission \\
ating room for surgical reduction & ( $>37$ degrees celsius) \\
may also be included. & Severe systemic injury \\
& Severe bleeding \\
& Pregnancy \\
& Coagulopathy \\
& Thrombocytopenia \\
& Known prior cardiac history \\
& Blood discrasia \\
& Pancreatitis \\
& Reynaud's syndrome \\
Cord transaction \\
Patients who are intubated and \\
sedated before initial examina- \\
tion by the neurosurgical team \\
Patients showing an improve- \\
ment in the neurologic exam \\
within $12 \mathrm{~h}$ from the injury
\end{tabular}

Abbreviations: GCS, Glasgow Coma Scale; ISNCSCI, International Standards for Neurological Classification of Spinal Cord Injury scale.

catheter (US Food and Drug Administration approved, Premarket Notification [501(k), K030421]; Alsius Corporation, Irvine, CA, USA) was inserted through the femoral vein soon after admission. Patients were cooled at a maximum rate $\left(2-2.5^{\circ} \mathrm{C}\right.$ per hour) until they reached target temperature $\left(T 33^{\circ} \mathrm{C}\right)$, which was maintained for $48 \mathrm{~h}$, and then re-warmed at $0.1^{\circ} \mathrm{C}$ per hour until normothermia $\left(T 37^{\circ} \mathrm{C}\right)$ was achieved. Patients were intubated and sedated using muscle relaxants as required by their clinical condition. Additional forms of sedation (benzodiazepines, dexmedetomidine, meperidine) were used to prevent shivering response. Serum electrolytes, coagulation studies and complete blood count (CBC) were performed daily. MAP was kept at $>85 \mathrm{~mm} \mathrm{Hg}$ and hematocrit at $\geqslant 30$. An orogastric/nasogastric tube was placed in each patient. Nutrition was provided per nutritional supplementation protocol. Deep venous thrombosis (DVT) /pulmonary embolism (PE) prophylaxis was initiated in all patients. At the time of CoolGard (Alsius Corporation, Irvine, CA, USA) removal, a venous Doppler ultrasound was performed in the lower limbs to evaluate the possibility of DVT. All the details of conducting the hypothermia protocol were previously published in Levi et al, $2009^{8}$

Patients underwent follow-up evaluations performed at acute care discharge, rehabilitation discharge, 6 weeks, 6 months and 1 year using ISNCSCI scale. The University of Miami Data Safety Monitoring Board evaluated hypothermia related potential complications, including infection, acute respiratory distress syndrome, DVT, pneumonia, atelectasis, electrolyte imbalance, septicemia, cardiac abnormalities (arrhythmias), thrombocytopenia, PE and death.

\section{RESULTS}

A total of 35 patients were enrolled: 8 females, 27 males (mean age $36.1 \pm 2.54$ years, range $18-65)$. The majority of patients had C5 or C6 injuries (15 and 10, respectively), while six had C4 and four had C7. Seventy-four percent (26 out of 35) of injuries were related to motor vehicle accidents, pedestrians hit by car or diving (Table 2) and were, for the most part, high-velocity injuries.

The mean $( \pm$ s.e.m.) time from injury to initiation of hypothermia was $7.76( \pm 1.09)$ hours. If we exclude four cases with delayed admission $(>18 \mathrm{~h})$, the average initiation time $(n=31)$ was 5.76 $( \pm 0.45)$ hours from the time of injury (Figure 1). After insertion of the CoolGard and initiation of hypothermia, the target temperature
Table 2 Demographics, mechanism and level of injury of 35 patients

\begin{tabular}{|c|c|c|c|c|c|}
\hline $\begin{array}{l}\text { Patient } \\
\text { ID no. }\end{array}$ & $\begin{array}{l}\text { Age (when } \\
\text { injured) }\end{array}$ & $\begin{array}{c}\text { Mechanism of } \\
\text { Injury }\end{array}$ & $\begin{array}{c}\text { Spinal } \\
\text { injury level }\end{array}$ & $\begin{array}{l}\text { Latest F/U } \\
\text { (months) }\end{array}$ & $\begin{array}{l}\text { ISNCSCI } \\
\text { at F/U }\end{array}$ \\
\hline 1 & 18 & Diving & $\mathrm{C} 4$ & 12 & A \\
\hline 2 & 20 & MVA & C4 & 12 & $A$ \\
\hline 3 & 55 & $\begin{array}{l}\text { Pedestrian hit } \\
\text { by car }\end{array}$ & C4 & 12 & C \\
\hline 4 & 21 & Diving & C4 & 12 & $A$ \\
\hline 5 & 21 & MVA & $\mathrm{C} 4$ & 12 & $C$ \\
\hline 6 & 53 & Fall & $\mathrm{C} 4$ & 12 & $B$ \\
\hline 7 & 62 & MVA & C5 & 12 & $B$ \\
\hline 8 & 16 & Fall & C5 & 12 & B \\
\hline 9 & 46 & MVA & C5 & 12 & $A$ \\
\hline 10 & 21 & Diving & C5 & 12 & $A$ \\
\hline 11 & 22 & Diving & C5 & 12 & $A$ \\
\hline 12 & 27 & Diving & C5 & 12 & $A$ \\
\hline 13 & 30 & Fall & C5 & 12 & A \\
\hline 14 & 22 & Fall & C5 & 12 & $D$ \\
\hline 15 & 51 & Fall & C5 & 12 & $A$ \\
\hline 16 & 38 & Diving & C5 & 12 & $A$ \\
\hline 17 & 27 & Diving & C5 & 12 & $A$ \\
\hline 18 & 39 & MVA & C5 & 12 & $A$ \\
\hline 19 & 32 & Fall & C5 & 12 & $A$ \\
\hline 20 & 23 & MVA & C5 & 12 & $D$ \\
\hline 21 & 39 & MVA & C5 & 12 & A \\
\hline 22 & 53 & MVA & C6 & 12 & C \\
\hline 23 & 20 & MVA & C6 & 12 & A \\
\hline 24 & 45 & Diving & C6 & 12 & D \\
\hline 25 & 29 & Diving & $\mathrm{C} 6$ & 12 & A \\
\hline 26 & 20 & Fall & $\mathrm{C} 6$ & 12 & $B$ \\
\hline 27 & 47 & MVA & $\mathrm{C} 6$ & 12 & $A$ \\
\hline 28 & 42 & MVA & C6 & 12 & $E$ \\
\hline 29 & 61 & MVA & C6 & 1.5 & $A$ \\
\hline 30 & 61 & Fall & C6 & 12 & C \\
\hline 31 & 23 & Fall & C6 & 12 & $B$ \\
\hline 32 & 65 & MVA & C7 & 12 & A \\
\hline 33 & 43 & $\begin{array}{l}\text { Pedestrian hit } \\
\text { by car }\end{array}$ & C7 & 6 & A \\
\hline 34 & 26 & MVA & C7 & 3 & D \\
\hline 35 & 45 & MVA & C7 & 3 & B \\
\hline
\end{tabular}

Abbreviation: F/U, follow up

was achieved in $<3 \mathrm{~h}(2.70 \pm 0.20)$. The duration of hypothermia at target temperature was $46.77( \pm 1.71)$ hours and total duration of cooling and re-warming was $113.55( \pm 5.10)$ hours.

All patients were complete ISNCSCI A on admission, but four converted to ISNCSCI B in $<24 \mathrm{~h}$ post injury. Fifteen of total 35 patients $(43 \%)$ improved at least one ISNCSCI grade at latest followup $11.01( \pm 0.48)$ months (Table 2$)$. In the group of patients $(n=31)$ with stable ISNCSCI A within first $24 \mathrm{~h}$ : six $(17.1 \%)$ converted to ISNCSCI B, three $(8.6 \%)$ to ISNCSCI C, two (5.7) to ISNCSCI D for a global conversion rate of $35.5 \%$ for stable ISNCSCI A patients in first $24 \mathrm{~h}$ (Figure 2). The neurological outcome in the remaining group of the four patients who converted to ISNCSCI B in $<24 \mathrm{~h}$ was the following: one $(2.9 \%)$ improved to ISNCSCI C, two $(5.7 \%)$ to ISNCSCI D and one (2.9\%) to ISNCSCI E (Figure 3). No correlation was found between improvement in ISNCSCI grade at latest followup and a shorter cooling time from injury: the majority of patients who did not improve (18 out of 20 ) received cooling in $<8 \mathrm{~h}$ (Figure 4). 


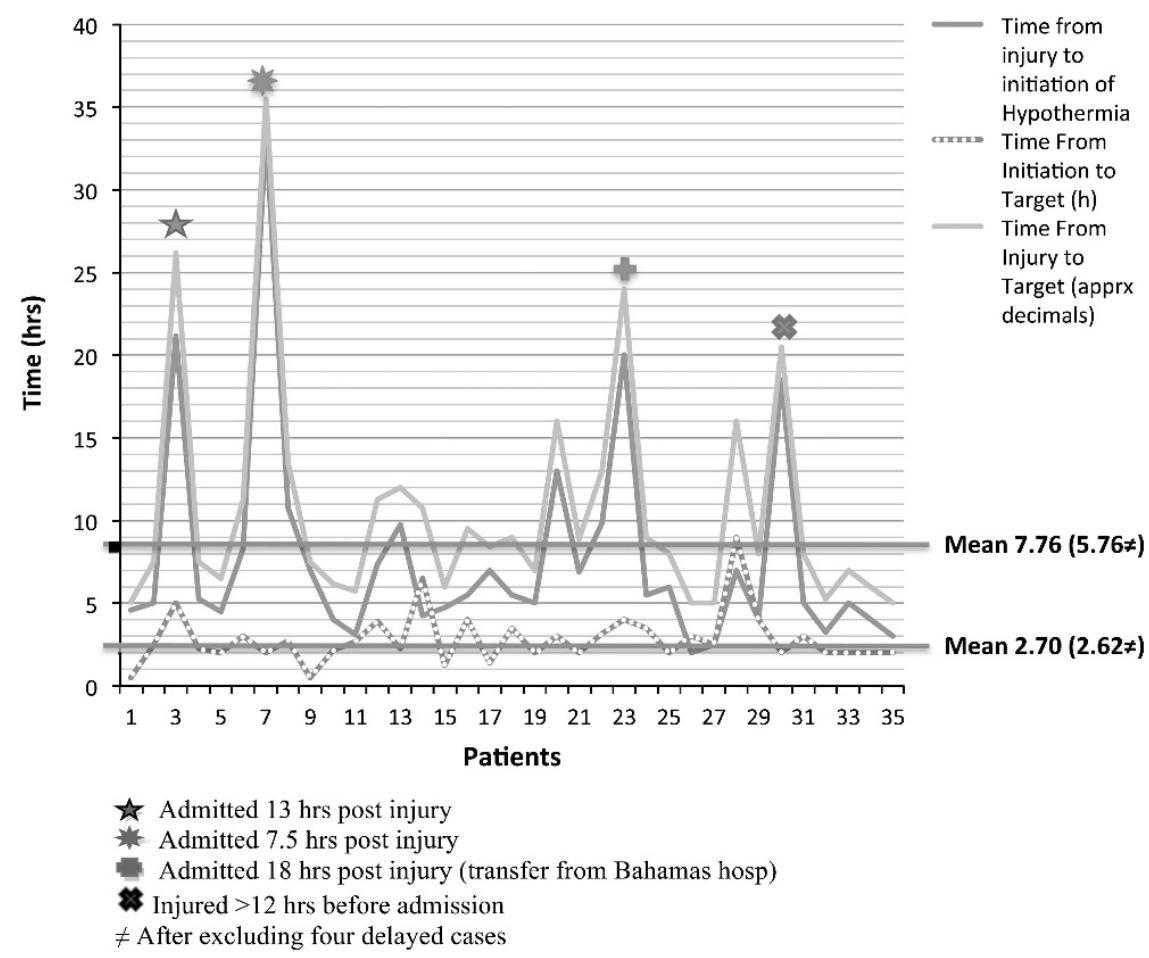

Figure 1 Timing of hypothermia administration in 35 patients.

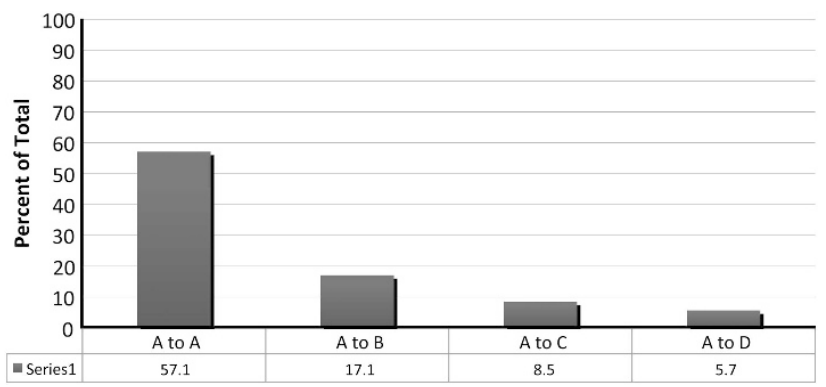

Figure 2 ISNCSCl outcome in 31 patients with initial and stable ISNCSCI A who did not improve within first $24 \mathrm{~h}$ from admission.

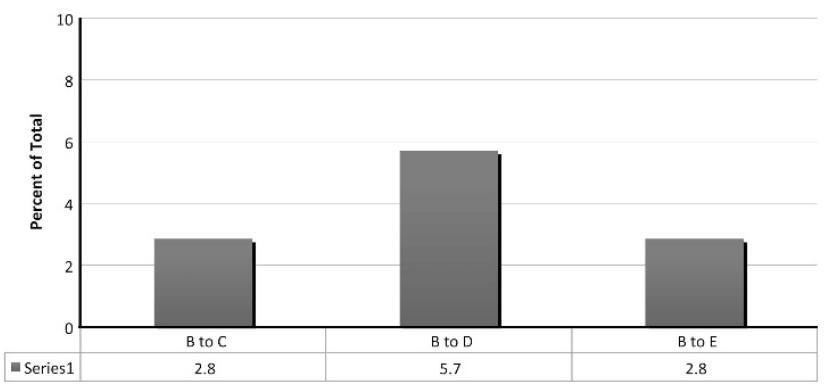

Figure 3 ISNCSCl outcome in four patients, who were converted to ISNCSCI B within $<24 \mathrm{~h}$ after admission.

All patients underwent surgery under conditions of hypothermia with no complications during or immediately after surgery related to hypothermia. Early decompression $(<24 \mathrm{~h})$ was done in majority of patients (29 out of $35 ; 83 \%)$. Thirty-eight percent of patients (11 out

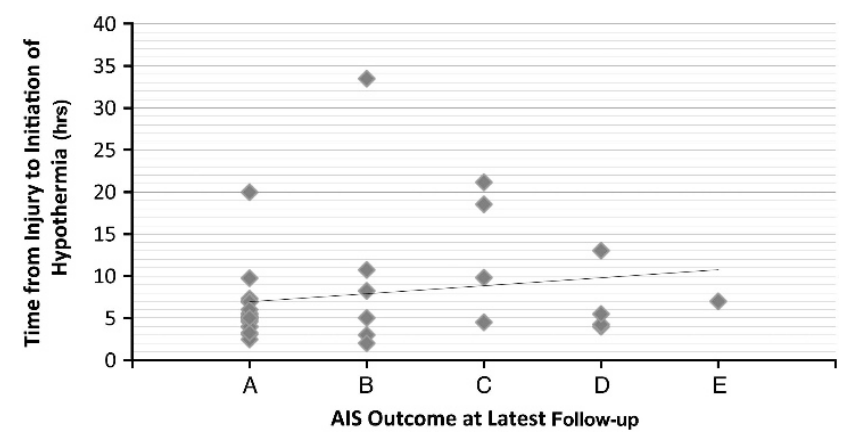

Figure 4 Time from injury to initiation of hypothermia vs ISNCSCI outcome.

of 29) with early decompression improved to ISNCSCI $>$ A at the latest follow-up vs $66.7 \%$ ( 4 out of 6 ) with $>24$ h decompression. Time from injury to surgical decompression for the improved patients $(n=15$ patients) was $16.4 \mathrm{~h}( \pm 6.08)$ vs $17.95 \mathrm{~h}( \pm 3.54)$ the group of non-improved patients ( $n=20$ patients).

We recorded all complications during the acute and follow-up care. Most complications were respiratory in origin: atelectasis in $83 \%$ $(n=29)$, pneumonia $60 \%(n=21)$, pleural effusion $54 \%(n=19)$, pulmonary edema $43 \%(n=15)$ and acute respiratory distress syndrome $11 \%(n=4)$ (Figure 5$)$. Two patients developed transient cardiac asystole: one-likely due to hypoxia and another after repositioning of a patient-that was shortly resolved without sequelae. A further reduction in heart rate with systemic hypothermia after SCI has been previously reported. ${ }^{8}$ Thirteen patients (37\%) developed urinary tract infections. Five thromboembolic complications (14\%) were observed: two PEs, one inferior vena cava clot, one DVT of femoral vein and one clot seen in a subclavian vein. All thromboembolic complications were well managed with 


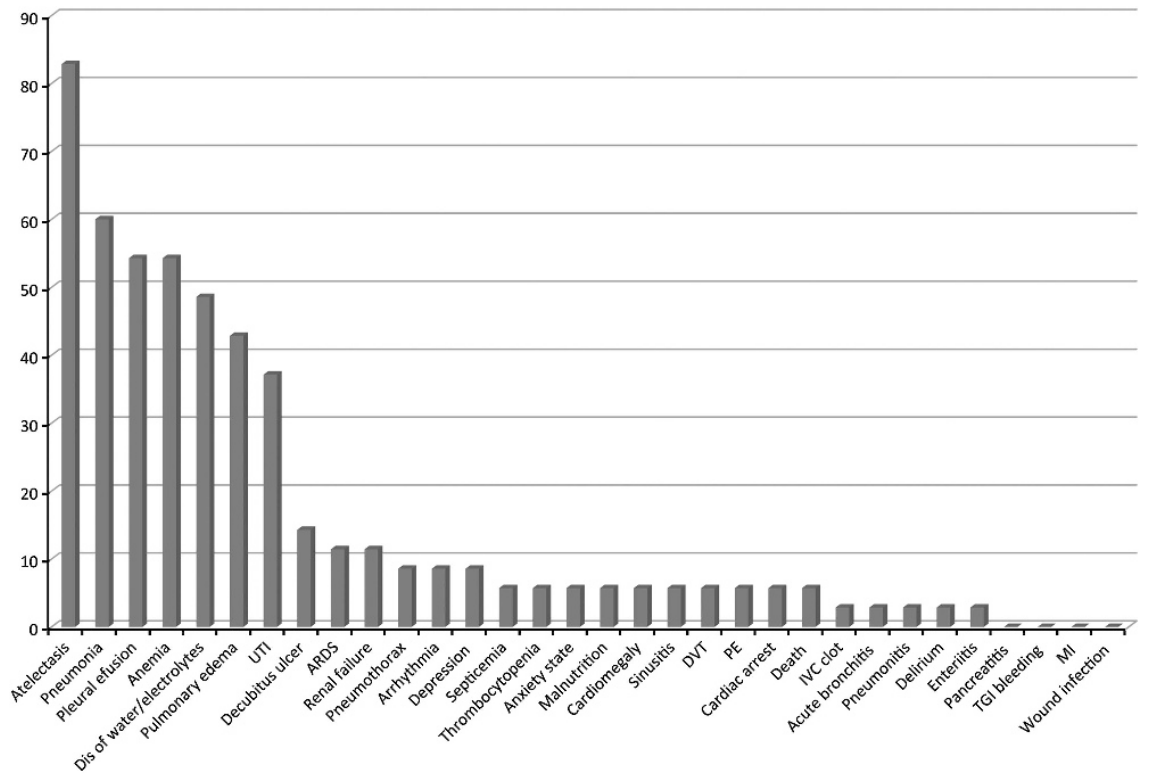

Figure 5 Percentage of complications in 35 patients recorded up to latest follow-up.

anticoagulants. Interestingly, in our first 14 patients receiving hypothermia - no patients developed thromboembolic complications; while in prospective 21 patients we observed five events (24\%). The clot within the subclavian and inferior vena cava were unrelated to the catheter. Also of note is that the first group received Lovenox (SanofiAventis, Bridgewater, NJ, USA) prophylaxis and the second Fragmin (Eisai Inc., Woodcliff Lake, NJ, USA) due to a hospital-wide mandatory change between the two studies. No patient died as a direct result of hypothermia. Two deaths were seen: one patient (aged 62 years, C5 injury) died of pneumonia 1 year post injury and another patient (aged 55 years, C5 injury) died of respiratory failure 2 months post injury.

\section{DISCUSSION}

\section{The timing of hypothermia}

Most promising strategies for a neuroprotection require early administration. The most well-known agent for SCI is methylprednisolone. ${ }^{9}$ The results of the multi-center trials suggest that early $(<8 \mathrm{~h})$ administration is required to observe a beneficial effect, and no effect was seen when late administration $(>8 \mathrm{~h})$ was included in the analysis. One of the secondary aims was to deliver systemic hypothermia as soon as possible upon admission. Although intravascular catheters for hypothermia are magnetic resonance imaging (MRI) compatible, allowing initiation of cooling before neuroimaging studies in the trauma ER, our 31 patients (excluding four delayed cases) received hypothermia in $5.76 \pm 0.45 \mathrm{~h}$ from injury. In prospective patient group $(n=21)$, hypothermia was initiated within a similar time frame post injury compared with the retrospective $(n=14)$ group ( 5.51 and $6.15 \mathrm{~h}$, respectively). ${ }^{8}$ Several factors are involved in these delays including transportation to trauma center, evaluation by the trauma team to rule out multi-system injury, imaging, consent process, insertion of the catheter, and so on. One can potentially decrease body temperature earlier by administering intravenous iced saline and applying cooling blankets in the field. Interestingly, when we looked at patients' improvement of at least one ISNCSCI grade or better and correlated with timing of hypothermia administration, there did not appear to be a clear benefit for early administration in this case series.

\section{Neurologic improvement after SCI}

The prospective patients revealed neurological improvement after hypothermia ( 9 out of $21-43 \%$ ) similar to the retrospective patients (6 out of $14-43 \%$ ) previously reported. ${ }^{7}$ The overall ISNCSCI conversion rates in our cervical SCI patients, who were ISNCSCI A on admission, was $43 \%$ ( 15 out of 35 ); even excluding those patients who converted from ISNCSCI A within $24 \mathrm{~h}$.

Thirty-five percent (11 out of 31 ) of the cervical ISNCSCI A patients improved. This supports our hypothesis that patients who received hypothermia demonstrate functional improvement, which compares favorably to historical data. According to three major SCI database control group studies, overall only $26.1 \%$ improved to ISNCSCI >A grade in cervical SCI population: $73.9 \%$ remained ISNCSCI A, $14.3 \%$ converted to ISNCSCI B, 5.6\% to ISNCSCI C, $6.2 \%$ to ISNCSCI D and none of the patients improves to ISNCSCI E level. ${ }^{10-12}$ The recent data from National Spinal Cord Injury Database $^{13}$ revealed higher spontaneous conversion rates after traumatic cervical SCI subjects: $30 \%$ of initial ISNCSCI grade A improved by 1 year, with $22 \%$ of them converting to ISNCSCI grade $\mathrm{B}$ or better by rehabilitation discharge. We had four prospective patients who improved to ISNCSCI B after $12 \mathrm{~h}$ but within $24 \mathrm{~h}$ after injury. They were enrolled into hypothermia protocol upon admission according to the inclusion criteria described above and received hypothermia within the first $6 \mathrm{~h}$. All these patients revealed high improvement rates, converting to ISNCSCI C, ISNCSCI D and ISNCSCI E (Figure 3) likely related to multiple factors, including early admission, expedient decompression, timely, as well as properly delivered cooling. Pre-reduction computed tomography (CT) and postoperative magnetic resonance imaging of the patient who improved to ISNCSCI E are shown (Figure 6).

\section{The timing of surgical decompression}

The optimal time for surgical decompression after SCI remains controversial. A meta-analysis concluded that class II evidence exists 

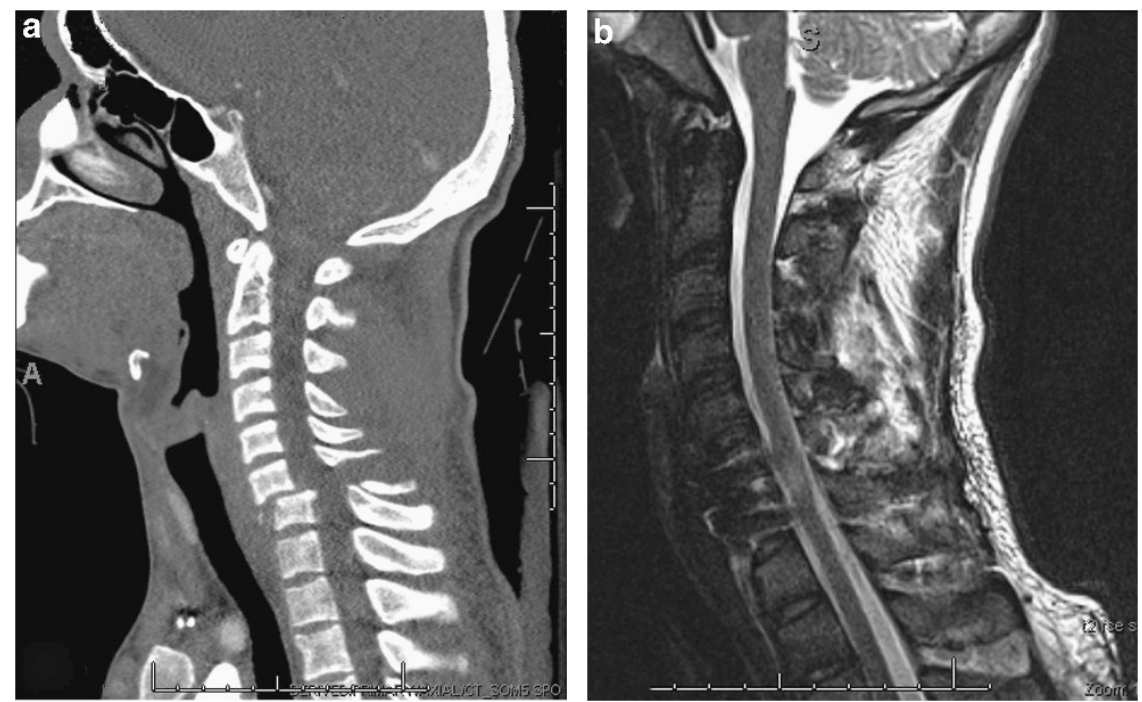

Figure 6 (a) Preop CT demonstrating a severe bilateral facet-fracture dislocation at C6-7. The patient underwent emergent cooling and reduction and fixation of the injury. (b) An MRI performed 4 days post injury demonstrates re-establishment of canal diameter and spinal cord decompression with intrinsic spinal cord change at the level of injury. The patient ultimately converted from ISNCSCI A to B within $24 \mathrm{~h}$ and ultimately ISNCSCI E at final follow-up.

that early surgery $(<24 \mathrm{~h})$ results in better neurological outcomes than delayed surgery $(>24 \mathrm{~h}){ }^{14}$ A recent evidence-based review recommended early intervention; although a number of studies indicated that patients who underwent early decompression had similar outcomes to those who had delayed decompression. ${ }^{15}$ In spite of unclear influence of early surgery on acute SCI outcome, the international spine community favors it based on the available clinical evidence and their own experience. The most recent multi-center, international, prospective cohort study in 313 cervical SCI patients from six North American centers concluded that $<24 \mathrm{~h}$ decompression is not only safe, but also improves neurological outcome (at least a two-grade ISNCSCI improvement) at 6-month follow-up. ${ }^{16}$ The average time of decompression between the improved and non-improved patients $(16.4 \mathrm{~h}$ vs $17.95 \mathrm{~h})$ in this study was not appreciably different. The majority of cases were decompressed in $<24 \mathrm{~h}$ and $38 \%$ of them improved vs $66.7 \%$ of patients improving in the $>24$-h group. The numbers of patients in both the groups are small ( 11 out of 29 vs 4 out of 6). The neurological improvement seen in our patients cannot be definitively ascribed to early surgery.

\section{Complications after SCI}

Atelectasis, pneumonia, pulmonary edema, pleural effusion, acute respiratory distress syndrome and even respiratory failure are common in a patient with complete (ISNCSCI A) high SCI without hypothermia treatment. The high incidence of respiratory complications in prospective patients corresponds to the data described elsewhere. ${ }^{7,8}$ Three cases of acute respiratory distress syndrome were likely related to aspiration of salt water due to drowning in one case and aspiration of orogastric fluid during resuscitation in another two cases. Paralyzed patients are already at high risk for thromboembolic complications without hypothermic treatment. The incidence of DVTs after SCI can vary from $5.3-38.3 \%$ in a recent meta-analysis study, ${ }^{17}$ and up to $43 \%$ when examined prospectively in 37 SCI patients. ${ }^{18}$ The mortality rate from DVT/ PE is $9.7 \%$ during the first year after SCI injury in patients who didn't receive hypothermia treatment. ${ }^{19}$ Patients with hypothermia treatment are potentially even in a higher risk group due to series of coagulation events in response to cooling and the presence of an intravascular catheter. A small study $(n=10)$ reported a $50 \%$ incidence of DVTs in cooled patients with traumatic brain injury. ${ }^{20}$ We were expecting a high number of thromboembolic complications in our cooled population, but we did not observe any DVT and PE in retrospective group $(n=14){ }^{7}$ Five thromboembolic complications were seen in the prospective patients $(n=21)$ : two DVT (one seen in a subclavian vein unrelated to the femoral coolguard catheter), two PE and one inferior vena cava clot. All hypothermic patients $(n=35)$ underwent Doppler screening for DVT after removal of the catheter and clinical screening for DVT and PE. They all received prophylaxis with low-molecular-weight heparin, together with compression stockings. The change in incidence may be related in part to the change in our method of DVT prophylaxis between the two groups (Lovenox vs Fragmin) as all other factors remained constant. In spite of the higher incidence in the prospective group, the overall incidence of thromboembolic complications in our hypothermic patients was similar to our prior experience and also with published data. Although hypothermia alters coagulation cascade, stasis, hypercoagulability, intimal injury and immobility related to the SCI are likely the major determining factors for thromboembolism in our patients. ${ }^{20}$

\section{Future perspectives}

To determine if therapeutic hypothermia results in neurological and possibly functional improvement after SCI, we are pursuing multicenter, prospective randomized trials, in cervical ISNCSCI A and ISNCSCI B patients. Significant improvement will be determined by a greater than 10 point difference in the mean change from baseline in ISNCSCI motor score when compared with normothermic patients, at 12 -month follow-up. ${ }^{10}$ Statistical analysis using the current data would require a sample size for randomization is 212 subjects ( 106 for each group).

\section{CONCLUSION}

The results are promising in terms of safety and improved functional outcome and to date represent the largest prospective series of cervical 
SCI patients treated by modest hypothermia described. A multicenter, prospective, randomized study is required to determine if systemic hypothermia for SCI should be a part of treatment for these patients for whom few treatment options exist.

\section{DATA ARCHIVING}

There were no data to deposit.

\section{CONFLICT OF INTEREST}

The authors declare no conflict of interest.

\section{ACKNOWLEDGEMENTS}

We received no external grant support for this study. We were not compensated nor have a financial interest in drugs, materials or devices described in this submission.

1 Dimar II JR, Shields CB, Zhang YP, Burke DA, Raque GH, Glassman SD. The role of directly applied hypothermia in spinal cord injury. Spine (Phila Pa 1976) 2000; 25: 2294-2302.

2 Kwon BK, Mann C, Sohn HM, Hilibrand AS, Phillips FM, Wang JC et al. Hypothermia for spinal cord injury. Spine J 2008; 8: 859-874.

3 Cappuccino A, Bisson LJ, Carpenter B, Marzo J, Dietrich WD, Cappuccino H. The use of systemic hypothermia for the treatment of an acute cervical spinal cord injury in a professional football player. Spine (Phila Pa 1976) 2010; 35: E57-E62.

4 Dietrich WD, Atkins CM, Bramlett HM. Protection in animal models of brain and spina cord injury with mild to moderate hypothermia. J Neurotrauma 2009; 26: 301-312.

5 Guest JD, Dietrich WD. Spinal cord ischemia and trauma. In: Tisherman SA and Sterz F (eds). Therapeutic Hypothermia. Springer: New York, 2005, pp 101-118.

6 Maybhate A, Hu C, Bazley FA, Yu Q, Thakor NV, Kerr CL et al. Potential long-term benefits of acute hypothermia after spinal cord injury: assessments with somatosensoryevoked potentials. Crit Care Med 2012; 40: 573-579.

7 Levi AD, Casella G, Green BA, Dietrich WD, Vanni S, Jagid J et al. Clinical outcomes using modest intravascular hypothermia after acute cervical spinal cord injury. Neurosurgery 2010; 66: 670-677.
8 Levi AD, Green BA, Wang MY, Dietrich D, Brindle T, Vanni S et al. The clinica application of modest hypothermia in the treatment of acute spinal cord injury. J Neurotrauma 2009; 26: 407-415.

9 Bracken MB, Shepard MJ, Holford TR, Leo-Summers L, Aldrich EF, Fazl M et al. Administration of methylprednisolone for 24 or 48 hours or tirilazad mesylate for 48 hours in the treatment of acute spinal cord injury. Results of the Third National Acute Spinal Cord Injury Randomized Controlled Trial. National Acute Spinal Cord Injury Study. JAMA 1997; 277: 1597-1604.

10 Steeves J, Lammertse D, Curt A, Fawcett JW, Tuszynski MH, Ditunno JF et al. Guidelines for the conduct of clinical trials for spinal cord injury as developed by the ICCP Panel: clinical trial outcome measures. Spinal Cord 2007; 45: 206-221.

11 Geisler FH, Coleman WP, Grieco G, Poonian DSygen Study Group. The Sygen multicenter acute spinal cord injury study. Spine (Phila Pa 1976) 2001; 26 S87-S98.

12 van Middendorp JJ, Hosman AJ, Pouw MH, Van de Meent HEM-SCI Study Group. ASIA impairment scale conversion in traumatic $\mathrm{SCl}$ : is it related with the ability to walk? A descriptive comparison with functional ambulation outcome measures in 273 patients. Spinal Cord 2009; 47: 555-560.

13 Marino RJ, Burns S, Graves DE, Leiby BE, Kirshblum S, Lammertse DP. Upper- and lower-extremity motor recovery after traumatic cervical spinal cord injury: an update from the national spinal cord injury database. Arch Phys Med Rehabil 2011; 92 369-375.

14 La Rosa G, Conti A, Cardali S, Cacciola F, Tomasello F. Does early decompression improve neurological outcome of spinal cord injured patients? Appraisal of the literature using a meta-analytical approach. Spinal Cord 2004; 42: 503-512.

15 Furlan JC, Noonan V, Cadotte DW, Fehlings MG. Timing of decompressive surgery of spinal cord after traumatic spinal cord injury: an evidence-based examination of preclinical and clinical studies. J Neurotrauma 2011; 28: 1371-1399.

16 Fehlings MG, Vaccaro A, Wilson JR, Singh A, W Cadotte D, Harrop JS et al. Early versus delayed decompression for traumatic cervical spinal cord injury: results of the surgical timing in Acute Spinal Cord Injury Study (STASCIS). PLoS One 2012; 7: e32037: (e-pub ahead of print 23 February, 2012).

17 Furlan JC, Fehlings MG. Role of screening tests for deep venous thrombosis in asymptomatic adults with acute spinal cord injury: an evidence-based analysis. Spine (Phila Pa 1976) 2007; 32: 1908-1916.

18 Chung SB, Lee SH, Kim ES, Eoh W. Incidence of deep vein thrombosis after spinal cord injury: a prospective study in 37 consecutive patients with traumatic or nontraumatic spinal cord injury treated by mechanical prophylaxis. J Trauma 2011; 71: 867-870; discussion 870-871.

19 Furlan JC, Fehlings MG. Cardiovascular complications after acute spinal cord injury: pathophysiology, diagnosis, and management. Neurosurg Focus 2008; 25: E13.

20 Simosa HF, Petersen DJ, Agarwal SK, Burke PA, Hirsch EF. Increased risk of deep venous thrombosis with endovascular cooling in patients with traumatic head injury. Am Surg 2007; 73: 461-464. 